\title{
Utilization of Digital Processing of the Optical Scanning Field View for Tip-Sample Distance Estimation during the Approach Procedure
}

\author{
A. SikORA ${ }^{a, *}$ AND E. BEDNARZ ${ }^{b}$ \\ ${ }^{a}$ Electrotechnical Institute, Division of Electrotechnology and Materials Science \\ M. Skłodowskiej-Curie 55/61, 50-369 Wrocław, Poland \\ ${ }^{b}$ Faculty of Microsystems Electronics and Photonics, Wrocław University of Technology \\ Z. Janiszewskiego 11/17, 50-372 Wrocław, Poland
}

\begin{abstract}
In this article we present the method of determining the tip-sample distance using advanced data processing of scanning field's optical picture. This feature can reduce both: the approach process time and the risk of damaging the tip or the sample. Experimental results will be also presented.
\end{abstract}

PACS numbers: 06.60.Sx, 07.05.Pj,07.79.--v

\section{Introduction}

Atomic force microscopy is one of the diagnostic techniques, which offers sub-micron resolution in both: lateral and vertical scales. It is possible due to observation of sophisticated interactions between the scanning tip and the surface. The tip which is developed using micromechanical processes, has typically the apex diameter in the range of $1-30 \mathrm{~nm}$. Therefore one can easily assume that such object is very fragile. It is essential that the tip should maintain its shape as long as possible in order to provide the best quality measurements results. The shape of the apex radius of the tip determines the way the sample is visualized, therefore this issue is one of important fields of the investigations [1-3]. It is known that before the sample is scanned, the tip must approach the surface and this process in one of most critical procedures in terms of risk of damaging the tip and the sample [4]. One desires to perform this process quickly in order to save the time and increase the throughput of the instrument. On the other hand, the approach should be performed carefully and slowly in order to avoid accidentally hitting the tip against the surface. Therefore the compromise must be achieved. The instruments are developed to provide safe approach automatically, however one can still see that available methods cannot guarantee really safe approach if the distance of hundreds of micrometers should be reduced within tens of seconds. Presented solution can make easier the coarse approach procedure to the distance of few micrometers, and eventually, slow approach can be started automatically.

* corresponding author; e-mail: sikora@iel.wroc.pl

\section{The method}

The method we proposed in this article uses CCD camera data processing. Such device together with an objective provides mostly the top-view of the scanning field as well as the cantilever. This is used for: alignment of the laser beam on the cantilever, positioning the tip in the area of interest and manual tip-sample distance reduction, which is far from being precise and reliable. By processing the video data we are able to determine if the object (the cantilever or surface) is in focal length of the objective. By raising or lowering the camera and finding two heights of its placement, where the cantilever and the surface are in focal length, we are able to calculate the distance between them. It should be mentioned that some commercially available systems offer such feature, however the evaluation if the object is in focal plane is made by the operator, which cannot provide high accuracy. The data processing we applied is performed as follows: 2-dimensional fast Fourier transformate (2D FFT), high-pass filtering, inverted fast Fourier transformate (IFFT) [5], measurement of the signal power histogram and finally, measurement of the peak's width on the histogram. The obtained value "F" correlates to the certain object imaging quality in terms of focus setting $[6,7]$. The window size of high-pass filter determines the quality of focus detection.

\section{The setup}

The tests were performed using the Innova system from Veeco Co. This instrument contains the CCD camera with $10 \times$ objective and PAL signal output. In order to process the signal, the frame-grabber PCI-1408 extension card from National Instruments was used. 


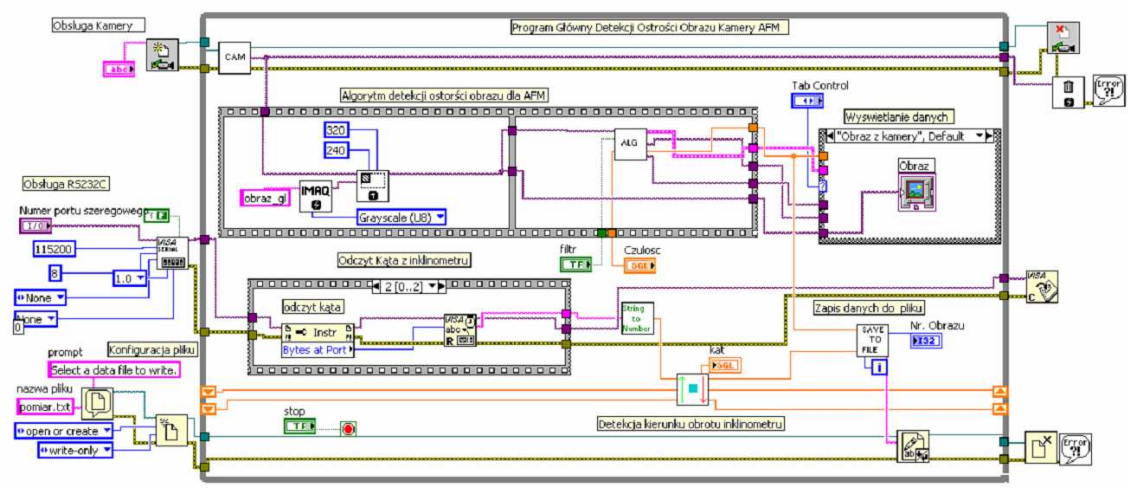

Fig. 1. Diagram of developed software.

The signal was processed using program developed in LabView environment. This tool has a large library of video data processing modules [8-10] which are useful in wide spectra of applications in scientific [11-13] and industry field [14]. The algorithm is presented in Fig. 1 and the program screenshot, in Fig. 2. The focus of the camera is controlled manually with the knob. Due to this fact, the position of the knob was detected with high-resolution digital-output inclinometer (ADIS16209 form Analog Devices). It allows to detect the rotation as small as $0.01^{\circ}$. The information about the knob position was transmitted to AUDC842 (Analog Devices) microcontroller via SPI interface, converted and sent to the computer using RS232 interface. Due to ability of AFM instrument of moving the cantilever by certain distance (calibrated in micrometers), we could obtain the distance-knob rotation. After performing the calibration (calculation of the knob rotation/camera height change), we were able to measure the distance between the surface and the cantilever.

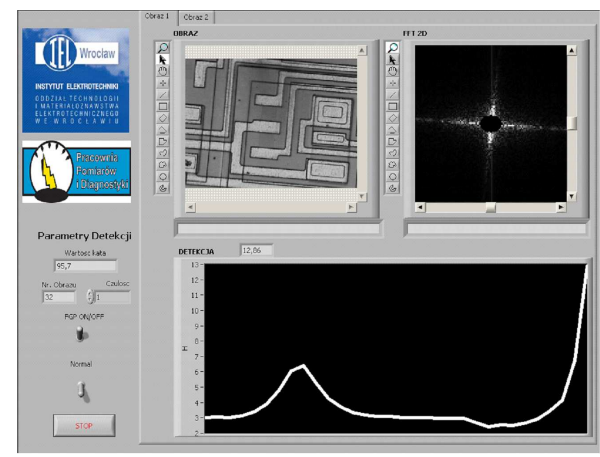

Fig. 2. Screenshot of developed data processing software.

\section{Experimental results}

The example of the measurement results is shown in Fig. 3. The curve showing the distance - "F" value, reaches two maxima: when the cantilever and the surface are in focal length from the objective. The influence of the resolution on the result repeatability and accuracy was evaluated by performing the measurement with $160 \times 120,320 \times 240$ and $640 \times 480$ resolution and for different distances (Table). As expected, the higher resolution allows to obtain better result, however requires more computation power.

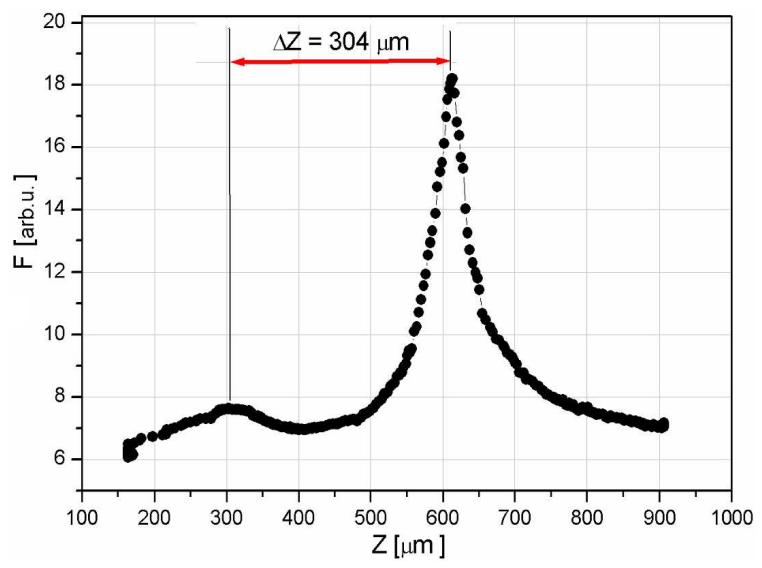

Fig. 3. The result of measurement with tip-sample distance estimation.

TABLE

The calibration factor calculation table for different cantilever-sample distances and picture resolutions.

\begin{tabular}{c|c|c|c}
\hline \hline \multirow{2}{*}{$\Delta Z[\mu \mathrm{m}]$} & \multicolumn{3}{|c}{ The calibration factor value } \\
\cline { 2 - 4 } & $160 \times 120$ & $320 \times 240$ & $640 \times 480$ \\
\hline 200 & 0.108 & 0.112 & 0.102 \\
300 & 0.115 & 0.108 & 0.101 \\
400 & 0.109 & 0.109 & 0.100 \\
500 & 0.113 & 0.111 & 0.101 \\
750 & 0.113 & 0.112 & 0.102 \\
1000 & 0.111 & 0.111 & 0.102 \\
mean value & 0.1115 & 0.1105 & 0.1013
\end{tabular}


Also the measurement for certain height was performed ten times. The difference was less than $2 \mu \mathrm{m}$, so one can consider this method as reliable and repetitive.

\section{Conclusions and outlook}

The approach procedure, if performed incorrectly can lead to damaging the tip and/or sample before starting the scanning process. This can cause serious problems with obtaining appropriate results of the measurements. We presented the method which was implemented in commercial setup without major hardware modifications, and allowed us to obtain quantitative information about the tip-sample distance before running the approach procedure. Therefore we could bring quickly the tip to distance of few $\mu \mathrm{m}$ from the surface. Such procedure reduces the time of operation significantly.

The presented method has a limitation, if transparent or semitransparent samples are considered. In such case the camera is unable to deliver a picture which would give satisfying calculation result. In many applications, however, this problem is negligible. Also mirror-like surfaces often do not reveal the features which can be useful for " $F$ " factor calculation. In this case, however, the reflection of the cantilever can be seen in doubled distance between the tip and sample. Further development of the method can resolve this problem. Using advanced algorithms with shape recognition can make the procedure more autonomous. Also implementing the stepper, in order to provide constant speed of the camera movement, will increase the accuracy. The application of presented solution can be particularly useful if extremely fragile tips are used (super sharp or nanotube-based tips).

\section{References}

[1] M. Kopycinska-Müller, R.H. Geiss, P. Rice, D.C. Hurley, Mater. Res. Soc. Symp. Proc. 838E, O10.16.1 (2005).

[2] A.G. Khurshudov, K. Kato, H. Koide, Wear 203, 22 (1997).

[3] H. Liu, M. Klonowski, D. Kneeburg, G. Dahlen, M. Osborn, T. Bao, J. Vac. Sci. Technol. B 23, 3090 (2005).

[4] K.H. Chung, Y.H. Lee, D.E. Kim, Ultramicroscopy 102, 161 (2005).

[5] W.K. Pratt, Digital Image Processing, Wiley, Hoboken, New York 2007.

[6] J.C. Russ, The Image Processing Handbook Fifth Edition, CRC Press, Boca Raton 2007.

[7] T.P. Zieliński, Digital signal processing, WKŁ, 2005 (in Polish).

[8] T. Klinger, Image Processing with Labview and IMAQ Vision, Prentice Hall PTR, Upper Saddle River 2003.

[9] NI VISION Concepts Manual, National Instruments, Austin, Texas 2008.

[10] NI VISION for Visual Basic User Manual, Austin, Texas 2007.

[11] S. Michalak, Utilisation of IMAQ Software Package's Functions in Program for Noise and Dark Current Analysis in Video Sensors, PWT, 2005 (in Polish).

[12] Q. Yua, H.H. Chenga, W.W. Chengb, X. Zhoub, Adv. Eng. Software 35, 527 (2004).

[13] N.I. Shaikh, V. Prabhu, Computers Industry 56, 777 (2005).

[14] E.N. Malamasa, E.G.M. Petrakisa, M. Zervakisa, L. Petitb, J.-D. Legatb, Image Vision Comput. 21, 171 (2003). 